\title{
THE NONLINEAR DIFFUSION LIMIT FOR GENERALIZED CARLEMAN MODELS: THE INITIAL-BOUNDARY VALUE PROBLEM
}

\author{
FRANÇOIS GOLSE AND FRANCESCO SALVARANI
}

\begin{abstract}
Consider the initial-boundary value problem for the 2-speed Carleman model of the Boltzmann equation of the kinetic theory of gases (see [T. Carleman, "Problèmes mathématiques dans la théorie cinétique des gaz", Almqvist-Wiksells, Uppsala, 1957]), set in some bounded interval with boundary conditions prescribing the density of particles entering the interval. Under the usual parabolic scaling, a nonlinear diffusion limit is established for this problem. In fact, the techniques presented here allow treating generalizations of the Carleman system where the collision frequency is proportional to the $\alpha$-th power of the macroscopic density, with $\alpha \in[-1,1]$.
\end{abstract}

\section{Carleman models and their Diffusion limits}

In the 1930's, Carleman proposed a model [1] describing the time evolution of a monodimensional gas composed of two species of particles that move at a constant speed $c>0$ in the $x$-direction. The number density at time $t$ and position $x$ of particles moving at speed $+c$ is denoted by $u=u(x, t)$ while that of particles moving at speed $-c$ is denoted $v=v(x, t)$. Carleman's system is

$$
\begin{aligned}
\partial_{t} u+c \partial_{x} u & =(u+v)(v-u), \\
\partial_{t} v-c \partial_{x} v & =(u+v)(u-v) .
\end{aligned}
$$

A very natural extension of the model, called the generalized Carleman model, involves a collision frequency that is proportional to some power of the macroscopic density $\rho=u+v$, as follows:

$$
\begin{aligned}
\partial_{t} u+c \partial_{x} u & =(u+v)^{\alpha}(v-u), \\
\partial_{t} v-c \partial_{x} v & =(u+v)^{\alpha}(u-v) .
\end{aligned}
$$

For $\alpha=1$ the original Carleman system is recovered, whereas $\alpha=0$ gives another remarkable system, known as the Goldstein-Taylor model [5, 21, that can be reduced to a damped wave equation (the telegrapher's equation). In the latter case, there is an explicit representation of the solution in terms of the standard Poisson: see [12], p. 56 for this formula, originally found by M. Kac. 
Other instances of kinetic models with a collision frequency whose dependence on the macroscopic density is other than linear can be found in Radiative Transfer: see for instance 13 .

The only nontrivial hydrodynamic limits of the Carleman model, generalized or not, are diffusion limits - linear or nonlinear. Indeed, local equilibria for those models are those densities for which $(u-v)(u+v)^{\alpha}=0$, implying $u=v$. Hence all local equilibria for those models have mean velocity 0 : in the language of the kinetic theory of gases, analogues for the system (11) of local Maxwellians have mean velocity 0 . Hence the limiting dynamics of the system can be observed only on a longer time scale that corresponds with a diffusion equation.

After setting $c=1$ without loss of generality, we consider the limit as $\epsilon \rightarrow 0^{+}$of the following scaled version of (2):

$$
\begin{aligned}
\epsilon^{2} \partial_{t} u_{\epsilon}+\epsilon \partial_{x} u_{\epsilon} & =\left(u_{\epsilon}+v_{\epsilon}\right)^{\alpha}\left(v_{\epsilon}-u_{\epsilon}\right), \\
\epsilon^{2} \partial_{t} v_{\epsilon}-\epsilon \partial_{x} v_{\epsilon} & =\left(u_{\epsilon}+v_{\epsilon}\right)^{\alpha}\left(u_{\epsilon}-v_{\epsilon}\right) .
\end{aligned}
$$

Defining the macroscopic mass density $\rho_{\epsilon}$ and the current $j_{\epsilon}$ by

$$
\rho_{\epsilon}=u_{\epsilon}+v_{\epsilon}, \quad j_{\epsilon}=\frac{u_{\epsilon}-v_{\epsilon}}{\epsilon},
$$

we put the system (3) in the form

$$
\begin{aligned}
\partial_{t} \rho_{\epsilon}+\partial_{x} j_{\epsilon} & =0, \\
\epsilon^{2} \partial_{t} j_{\epsilon}+\partial_{x} \rho_{\epsilon} & =-2 \rho_{\epsilon}^{\alpha} j_{\epsilon} .
\end{aligned}
$$

Then, we prove that the term $\epsilon^{2} \partial_{t} j_{\epsilon}$ is negligeable in the vanishing $\epsilon$ limit, and show that the limiting density is governed by the following nonlinear diffusion equation

$$
\partial_{t} \rho=\frac{1}{2} \partial_{x x}\left(\frac{\rho^{1-\alpha}}{1-\alpha}\right),
$$

for $\alpha \in[-1,1)$, while the case $\alpha=1$ leads to

$$
\partial_{t} \rho=\frac{1}{2} \partial_{x x} \ln \rho .
$$

Several result on this problem have been obtained over the last thirty years.

In particular, existence and uniqueness for the solution of the initialboundary value problem of (1) have been proven by Fitzgibbon [3] - see also [7] for the same problem on the infinite line, and [16], 17] and [20] for more information concerning the large time behavior of the solutions 1 .

Several authors (among others Kurtz [8], McKean [12, Fitzgibbon [3], Pulvirenti and Toscani [15, P.-L. Lions and Toscani 9], Marcati and Rubino 11, Donatelli and Marcati [2], Salvarani and Vázquez [19]) have considered the relaxation problem for the Carleman system or generalizations thereof.

\footnotetext{
${ }^{1}$ We are grateful to L. Tartar for indicating the references [7] and [16], 17].
} 
All these papers on the diffusion limit for kinetic models deal with the initial value problem on the infinite line, or with the initial-boundary value problem with specular or periodic conditions at the boundary.

Establishing the diffusion limit for Carleman type models (generalized or not) in a bounded domain with non homogeneous boundary conditions is more difficult. Indeed, mimicking the proofs of the diffusion limit in the cases mentioned above fails to produce a uniform bound on the current $j_{\epsilon}$ in the case of a nonhomogeneous boundary value problem.

To the best of our knowledge, the only available convergence proof for an initial-boundary value problem with very general boundary conditions at the time of this writing is in [18] for the Goldstein-Taylor model. Unfortunately, the proof in [18] uses extensively the linear nature of the problem coming from the assumption $\alpha=0$, and does not seem to be extendible to nonlinear models.

The present work establishes the diffusion limit for all Carleman systems (2) with $\alpha \in[-1,1]$, in a bounded domain $\Omega=(0,1)$, with boundary conditions imposing the density of particles entering the domain $\Omega$

$$
\begin{aligned}
& u_{\epsilon}(t, 0)=\varphi^{-}(t), \quad t>0, \\
& v_{\epsilon}(t, 1)=\varphi^{+}(t), \quad t>0 \text {. }
\end{aligned}
$$

We have restricted our analysis to the case $|\alpha| \leq 1$, as it leads to a unified treatment based on the dissipative nature of the problem.

The new ingredient in the present paper is a uniform estimate bearing on some notion of relative entropy of the solution $\left(u_{\epsilon}, v_{\epsilon}\right)$ with respect to a wellchosen profile that satisfies the boundary conditions expected to hold in the vanishing $\epsilon$ limit. By failing to introducing this profile, previous attempts to establishing the nonlinear diffusion limit in the case of nonhomogeneous data fell short of obtaining uniform bounds on the current $j_{\epsilon}$, except in the particular case $\varphi^{+}(t)=\varphi^{-}(t)=$ Const .

This idea of using the relative entropy with respect to some adequate profile in order to match nontrivial boundary conditions can be used on more complicated models. In the introduction above, we already mentioned that the generalized Carleman equations are somewhat analogous to more complicated models appearing in Radiative Transfer, for which the nonlinear diffusion approximation is known under the name of "Rosseland approximation". We shall apply the method presented here to these more complicated models in a forthcoming paper [6].

\section{MAin RESUlts}

Before stating the convergence theorem that is the main result in this paper, we recall some background on the Carleman systems.

2.1. Existence and uniqueness theory for Carleman systems. The class of initial and boundary data for the Carleman systems considered in this paper, which we henceforth call "admissible data", is defined below. 
Definition 2.1. Consider the system (3) with $|\alpha| \leq 1$ posed for $(t, x) \in$ $(0, T) \times(0,1)$, with boundary conditions (8) and initial condition

$$
\begin{array}{ll}
u_{\epsilon}(0, x)=u^{i n}(x), & 0<x<1, \\
v_{\epsilon}(0, x)=v^{i n}(x), & 0<x<1 .
\end{array}
$$

The initial data $\left(u^{i n}, v^{i n}\right)$ and the boundary data $\left(\varphi^{-}, \varphi^{+}\right)$are said to be admissible on the time interval $(0, T)$ if and only if

(1) $\varphi^{ \pm} \in W^{1, \infty}([0, T])$ and $\varphi^{ \pm}>0$ on $(0, T)$, while

(2) $u^{\text {in }}$ and $v^{\text {in }} \in L^{3}(0,1)$ and $u^{i n}, v^{\text {in }} \geq 0$ a.e. in $(0,1)$.

The existence and uniqueness of a nonnegative solution for the original Carleman model is well-known. The following result is a standard generalization of Fitzgibbon's in [4]:

Theorem 2.2. Consider the generalized Carleman model (2) for $|\alpha| \leq 1$ in the domain $\Omega=(0,1)$ with boundary condition (8) and initial condition (9). Assume that the initial and boundary data $\left(u^{i n}, v^{i n}\right)$ and $\varphi^{ \pm}$are admissible. Then there exists a unique nonnegative generalized solution $(u, v)$ of (2) in $C\left([0, T] ; L^{1}(0,1) \times L^{1}(0,1)\right)$.

Proof. It is well known that the unbounded operator

$$
B_{\alpha}(u, v)=\left(-\partial_{x} u+(u+v)^{\alpha}(v-u), \partial_{x} v+(u+v)^{\alpha}(u-v)\right)
$$

on $L^{1}(0,1) \times L^{1}(0,1)$ with domain

$$
\mathcal{D}\left(B_{\alpha}\right)=\left\{(u, v) \in W^{1,1}(0,1) \times W^{1,1}(0,1) \mid u(0)=0 \text { and } v(1)=0\right\}
$$

is dissipative (see, for example, [9, 19]). The existence and uniqueness of the solution $(u, v)$ easily follows from the same method as in [4].

2.2. Uniqueness theory for the nonlinear diffusion equation. Next we consider the target, nonlinear diffusion equation (6) (or (7) when $\alpha=1$ ).

Definition 2.3. Consider the nonlinear diffusion equation (6) or (7) when $\alpha=1$ with Dirichlet boundary conditions

$$
\rho(t, 0)=2 \varphi^{-}, \quad \rho(t, 1)=2 \varphi^{+}, \quad t \in(0, T),
$$

and initial condition

$$
\rho(0, x)=\rho_{0}(x), \quad x \in(0,1) .
$$

We call these data admissible if and only if

(1) for $\alpha \in[-1,1), \rho_{0} \geq 0$ belongs to $L^{3}((0,1))$ while $\varphi^{ \pm} \geq 0$ is in $W^{1, \infty}([0, T])$

(2) for $\alpha=1$, same assumptions except that $\varphi^{ \pm}>0$.

The notion of admissible weak solution of the nonlinear diffusion equations (6) or (7) being less obvious than in the case of the Carleman model, we recall it below. 
Definition 2.4. An admissible weak solution of (6) or (17) with Dirichlet boundary conditions

$$
\rho(t, 0)=2 \varphi^{-}, \quad \rho(t, 1)=2 \varphi^{+}, \quad t \in(0, T)
$$

and initial condition

$$
\rho(0, x)=\rho_{0}(x), \quad x \in(0,1)
$$

for admissible data $\left(\rho_{0}, \varphi^{ \pm}\right)$is a nonnegative element of $L^{2}((0, T) \times(0,1))$ such that $\partial_{x} \rho^{1-\alpha} \in L^{2}((0, T) \times(0,1))$ if $\alpha \neq 1$ while $\ln \rho \in L_{l o c}^{1}((0, T) \times(0,1))$ with $\partial_{x} \ln \rho \in L^{2}((0, T) \times(0,1))$ if $\alpha=1$, that satisfies (10) for a.e. $t \in$ $(0, T)$, together with the identities

$$
\int_{0}^{T} \int_{0}^{1}\left(\rho \partial_{t} \phi-\frac{1}{2(1-\alpha)} \partial_{x} \rho^{1-\alpha} \partial_{x} \phi\right)(t, x) d x d t+\int_{0}^{1} \rho_{0}(x) \phi(x, 0) d x d t=0,
$$

for all $\phi \in C([0, T] \times[0,1]) \cap H^{1}([0, T] \times[0,1])$ vanishing in $x=0, x=1$ and $t=T$ if $\alpha \neq 1$, whereas, for $\alpha=1$,

$$
\int_{0}^{T} \int_{0}^{1}\left(\rho \partial_{t} \phi-\frac{1}{2} \partial_{x} \ln \rho \partial_{x} \phi\right)(t, x) d x d t+\int_{0}^{1} \rho_{0}(x) \phi(x, 0) d x d t=0 .
$$

Notice that, in what follows, we only need to know that the boundary value problem for (6) or (7) has at most one admissible solution, as the existence of such solutions will result from that of a solution of the generalized Carleman model in the hydrodynamic limit. The proof of the following lemma is classical:

Lemma 2.5. For each set of admissible data $\left(\rho_{0}, \varphi^{ \pm}\right)$, the nonlinear diffusion equation (6) for $-1 \leq \alpha<1$ or (7) if $\alpha=1$ has at most one admissible solution.

2.3. The convergence result. The main result in the present paper is the following convergence theorem.

Theorem 2.6. Let $\left(u^{i n}, v^{i n}, \varphi^{ \pm}\right)$be admissible data for the generalized Carleman system on the time interval $[0, T]$. For each $\epsilon>0$, let $\left(u_{\epsilon}, v_{\epsilon}\right)$ be the solution of the scaled Carleman system (13) with initial condition (19) and boundary data (8).

Then, in the limit as $\epsilon \rightarrow 0$, the macroscopic density

$$
\rho_{\epsilon}=u_{\epsilon}+v_{\epsilon} \rightarrow \rho \quad \text { in } L_{l o c}^{2}([0, T] \times[0,1])
$$

where $\rho$ is the generalized solution of (6) if $\alpha \in[-1,1)$ or of (7) if $\alpha=1$, with initial and boundary conditions

$$
\begin{gathered}
\rho(x, 0)=u^{i n}(x)+v^{i n}(x), \quad x \in(0,1), \\
\rho(t, 0)=2 \varphi^{-}, \quad \rho(t, 1)=2 \varphi^{+}, \quad t \in(0, T) .
\end{gathered}
$$




\section{UNIFORM BOUNDS ON THE DENSITY AND CURRENT}

As mentioned in the introduction, the uniform (in $\epsilon$ ) bounds on the density and current are consequences of the equation that is satisfied by some notion of relative entropy, which we shall introduce below.

From now on, we set

$$
\nu=\max \left(\left\|\varphi^{+}\right\|_{W^{1, \infty}(0, T)},\left\|\varphi^{-}\right\|_{W^{1, \infty}(0, T)}\right)
$$

and

$$
\varphi_{m}=\inf \left\{\varphi^{ \pm}(t) \mid 0 \leq t \leq T\right\}>0 .
$$

We begin with some elementary background on convex functions.

3.1. A family of convex functions. Let $\phi: \mathbf{R}_{+} \rightarrow \mathbf{R}$ be a convex function that is $C^{2}$ on $\mathbf{R}_{+}^{*}$, and that satisfies

$$
\phi_{d}^{\prime}(0) \leq 0
$$

and

$$
\frac{\phi(y)}{y} \rightarrow+\infty \text { as } y \rightarrow+\infty \text {. }
$$

Define the Legendre dual $\phi^{*}$ of $\phi$ by

$$
\phi^{*}(\xi)=\sup _{y \geq 0}(\xi y-\phi(y)) .
$$

This definition clearly implies that

$$
\xi y \leq \phi(y)+\phi^{*}(\xi) \text { for each } y, \xi \in \mathbf{R}_{+} .
$$

This inequality is a generalization of Young's classical inequality for

$$
\phi(y)=\frac{y^{p}}{p} \text { and } \phi^{*}(\xi)=\frac{\xi^{p^{\prime}}}{p^{\prime}}
$$

where $p$ and $p^{\prime}$ are dual exponents in the sense of Hölder's inequality:

$$
p \in[1, \infty) \text { and } p^{\prime}=\frac{p}{p-1} .
$$

More specifically, we shall use the family of convex functions below:

a) for $\beta \in[-1,1)$, the function $\phi_{\beta}$ is given by

$$
\phi_{\beta}(y)=\frac{1}{2-\beta} y^{2-\beta}, \quad y \geq 0 ;
$$

b) for $\beta=1$, the function $\phi_{1}$ is given by

$$
\phi_{1}(y)=y \log y \text { and } \phi_{1}(0)=0, \quad y>0 .
$$

Straightforward computations show that

$$
\phi_{\beta}^{*}(\xi)=\frac{1-\beta}{2-\beta} \xi^{\frac{2-\beta}{1-\beta}}
$$

while

$$
\phi_{1}^{*}(\xi)=e^{\xi-1}
$$

for each $\xi>0$ and $\beta \in[-1,1)$. 
With $\nu$ as in (16), for each $\beta \in[-1,1]$, we set

$$
\Phi_{\beta}(y)=\phi_{\beta}(y)+\phi_{\beta}^{*}\left(\phi_{\beta}^{\prime}(\nu)+1\right), \quad y \geq 0 .
$$

Young's inequality entails

$$
\Phi_{\beta}(y) \geq\left(\phi_{\beta}^{\prime}(\nu)+1\right) y, \quad y \geq 0,
$$

and since $\Phi_{\beta}^{\prime}=\phi_{\beta}^{\prime}$, one has

$$
\Phi_{\beta}(y)-\Phi_{\beta}^{\prime}(\nu) y \geq y, \quad \beta \in[-1,1], y \geq 0 .
$$

3.2. The relative entropy and entropy production rate. Our bounds on the Carleman system involve the notion of relative entropy with respect to some suitable profile that satisfies the same boundary conditions as the solution of the limiting diffusion equation. There are many possible ways of choosing this profile. When the boundary data are constant, a logical choice would be to pick the stationary solution of the diffusion equation with those boundary data. In the case of time dependent boundary conditions, this choice is less natural, and we simply define this profile to be the convex combination of boundary data:

$$
f(t, x):=(1-x) \varphi^{-}(t)+x \varphi^{+}(t) .
$$

Given $\Phi: \mathbf{R}_{+} \rightarrow \mathbf{R}$, a $C^{2}$ convex function, we define the relative entropy of the 2-velocity density $(u, v) \equiv(u(x), v(x)) \in\left(\mathbf{R}_{+}\right)^{2}$ to be

$$
H_{\beta}[u, v \mid f]=\int_{0}^{1}\left[\Phi_{\beta}(u)+\Phi_{\beta}(v)-2 \Phi_{\beta}(f)-\Phi_{\beta}^{\prime}(f)(u+v-2 f)\right](x) d x
$$

and the entropy production rate as

$$
P_{\beta}[u, v]=\int_{0}^{1}\left(\Phi_{\beta}^{\prime}(u)-\Phi_{\beta}^{\prime}(v)\right)(u+v)^{\alpha}(u-v)(x) d x \geq 0
$$

Assuming that $\left(u_{\epsilon}, v_{\epsilon}\right)$ is a solution of the scaled Carleman problem (3), we next determine the equation governing the evolution of $H_{\beta}\left[u_{\epsilon}, v_{\epsilon} \mid f\right]$. Multiplying each side of the first equation in (3) by $\Phi_{\beta}^{\prime}\left(u_{\epsilon}\right)$ and each side of the second equation in (3) by $\Phi_{\beta}^{\prime}\left(v_{\epsilon}\right)$, one finds, upon adding both resulting equalities and integrating in $x$ over $[0,1]$ :

$$
\begin{aligned}
\frac{d}{d t} \int_{0}^{1}\left(\Phi_{\beta}\left(u_{\epsilon}\right)+\Phi_{\beta}\left(v_{\epsilon}\right)\right)(t, x) d x+\frac{1}{\epsilon} \int_{0}^{1} \partial_{x}\left(\Phi_{\beta}\left(u_{\epsilon}\right)\right. & \left.-\Phi_{\beta}\left(v_{\epsilon}\right)\right)(t, x) d x \\
& =-\frac{1}{\epsilon^{2}} P_{\beta}\left[u_{\epsilon}, v_{\epsilon}\right]
\end{aligned}
$$


It is actually more convenient to rearrange this equality as follows:

$$
\begin{array}{r}
\frac{d}{d t} \int_{0}^{1}\left(\Phi_{\beta}\left(u_{\epsilon}\right)+\Phi_{\beta}\left(v_{\epsilon}\right)-\Phi_{\beta}^{\prime}(f)\left(u_{\epsilon}+v_{\epsilon}\right)\right)(t, x) d x \\
+\frac{1}{\epsilon} \int_{0}^{1} \partial_{x}\left(\Phi_{\beta}\left(u_{\epsilon}\right)-\Phi_{\beta}\left(v_{\epsilon}\right)-\Phi_{\beta}^{\prime}(f)\left(u_{\epsilon}-v_{\epsilon}\right)\right)(t, x) d x \\
=-\frac{1}{\epsilon^{2}} P_{\beta}\left[u_{\epsilon}, v_{\epsilon}\right]-\frac{1}{\epsilon} \int_{0}^{1} \partial_{x} \Phi_{\beta}(f)\left(u_{\epsilon}-v_{\epsilon}\right)(t, x) d x \\
+\int_{0}^{1} \partial_{t} \Phi_{\beta}(f)\left(u_{\epsilon}+v_{\epsilon}\right)(t, x) d x .
\end{array}
$$

Observe that

$$
\begin{array}{r}
\frac{1}{\epsilon} \int_{0}^{1} \partial_{x}\left(\Phi_{\beta}\left(u_{\epsilon}\right)-\Phi_{\beta}\left(v_{\epsilon}\right)-\Phi_{\beta}(f)\left(u_{\epsilon}-v_{\epsilon}\right)\right)(t, x) d x \\
=\frac{1}{\epsilon}\left[\Phi_{\beta}\left(u_{\epsilon}\right)-\Phi_{\beta}(f)-\Phi_{\beta}^{\prime}(f)\left(u_{\epsilon}-f\right)\right]_{0}^{1} \\
-\frac{1}{\epsilon}\left[\Phi_{\beta}\left(v_{\epsilon}\right)-\Phi_{\beta}(f)-\Phi_{\beta}^{\prime}(f)\left(v_{\epsilon}-f\right)\right]_{0}^{1} \\
=\frac{1}{\epsilon}\left(\Phi_{\beta}\left(u_{\epsilon}\right)-\Phi_{\beta}(f)-\Phi_{\beta}^{\prime}(f)\left(u_{\epsilon}-f\right)\right)(t, 1) \\
+\frac{1}{\epsilon}\left(\Phi_{\beta}\left(v_{\epsilon}\right)-\Phi_{\beta}(f)-\Phi_{\beta}^{\prime}(f)\left(v_{\epsilon}-f\right)\right)(t, 0)
\end{array}
$$

since

$$
u_{\epsilon}(t, 0)=f(t, 0) \text { and } v_{\epsilon}(t, 1)=f(t, 1) .
$$

By convexity of $\Phi_{\beta}$, both terms in the last right hand side of (23) are nonnegative; hence

$$
\frac{1}{\epsilon} \int_{0}^{1} \partial_{x}\left(\Phi_{\beta}\left(u_{\epsilon}\right)-\Phi_{\beta}\left(v_{\epsilon}\right)-\Phi_{\beta}^{\prime}(f)\left(u_{\epsilon}-v_{\epsilon}\right)\right)(t, x) d x \geq 0 .
$$

Next, we formulate the equality (22) in terms of the relative entropy $H_{\beta}\left[u_{\epsilon}, v_{\epsilon} \mid f\right]$ as follows:

$$
\begin{array}{r}
\frac{d}{d t} H_{\beta}\left[u_{\epsilon}, v_{\epsilon} \mid f\right]+\frac{1}{\epsilon^{2}} P_{\beta}\left[u_{\epsilon}, v_{\epsilon}\right] \leq-\frac{1}{\epsilon} \int_{0}^{1} \partial_{x} \Phi_{\beta}(f)\left(u_{\epsilon}-v_{\epsilon}\right)(t, x) d x \\
+\int_{0}^{1} \partial_{t} \Phi_{\beta}^{\prime}(f)\left(u_{\epsilon}+v_{\epsilon}\right)(t, x) d x+\int_{0}^{1} \Phi_{\beta}^{\prime \prime}(f) \partial_{t} f^{2}(t, x) d x .
\end{array}
$$

We estimate the first term on the right hand side of the inequality (25) in terms of the current $j_{\epsilon}=\frac{1}{\epsilon}\left(u_{\epsilon}-v_{\epsilon}\right)$, as follows: for each $\gamma \in(0,1)$, one has (26)

$$
-\frac{1}{\epsilon} \int_{0}^{1} \partial_{x} \Phi_{\beta}(f)\left(u_{\epsilon}-v_{\epsilon}\right)(t, x) d x \leq \frac{\gamma}{2} \int_{0}^{1} j_{\epsilon}(t, x)^{2} d x+\frac{1}{2 \gamma}\left\|\partial_{x} \Phi_{\beta}(f)(t, x)\right\|_{L^{\infty}}^{2} .
$$

Then, the inequality (19) satisfied by $\Phi_{\beta}$ implies that

$$
\Phi_{\beta}(y)-\Phi_{\beta}^{\prime}(f(t, x)) y \geq \Phi_{\beta}(y)-\Phi_{\beta}^{\prime}(\nu) y \geq y
$$


for each $y \geq 0$. Indeed

$$
f(t, x) \leq \max \left(\left\|\varphi^{-}\right\|_{L^{\infty}(0, T)},\left\|\varphi^{+}\right\|_{L^{\infty}(0, T)}\right)
$$

so that

$$
\Phi_{\beta}^{\prime}(f(t, x)) \leq \Phi_{\beta}^{\prime}(\nu)
$$

since $\Phi_{\beta}^{\prime}$ is nondecreasing, $\Phi_{\beta}$ being convex. Because of (27), one has (28)

$$
\begin{aligned}
\int_{0}^{1} \partial_{t} \Phi_{\beta}(f) & \left(u_{\epsilon}+v_{\epsilon}\right)(t, x) d x \\
& \leq \int_{0}^{1}\left|\partial_{t} \Phi_{\beta}^{\prime}(f)\right|\left[\Phi_{\beta}\left(u_{\epsilon}\right)+\Phi_{\beta}\left(v_{\epsilon}\right)-\Phi_{\beta}^{\prime}(f)\left(u_{\epsilon}+v_{\epsilon}\right)\right](t, x) d x \\
& \leq\left\|\partial_{t} \Phi_{\beta}^{\prime}(f)\right\|_{L^{\infty}}\left(H_{\beta}\left[u_{\epsilon}, v_{\epsilon} \mid f\right]+2\left\|\Phi_{\beta}(f)-\Phi_{\beta}^{\prime}(f) f\right\|_{L^{\infty}}\right) .
\end{aligned}
$$

With this estimate for the second term on the right hand side of (25), we recast this inequality as

$$
\begin{array}{r}
\frac{d}{d t} H_{\beta}\left[u_{\epsilon}, v_{\epsilon} \mid f\right]+\frac{1}{\epsilon^{2}} P_{\beta}\left[u_{\epsilon}, v_{\epsilon}\right] \leq \frac{\gamma}{2} \int_{0}^{1} j_{\epsilon}(t, x)^{2} d x+\left\|\partial_{t} \Phi_{\beta}^{\prime}(f)\right\|_{L^{\infty}} H_{\beta}\left[u_{\epsilon}, v_{\epsilon} \mid f\right] \\
+\frac{1}{2 \gamma}\left\|\partial_{x} \Phi_{\beta}(f)\right\|_{L^{\infty}}^{2}+\left\|\Phi_{\beta}^{\prime \prime}(f) \partial_{t} f^{2}\right\|_{L^{\infty}} \\
+2\left\|\partial_{t} \Phi_{\beta}^{\prime}(f)\right\|_{L^{\infty}}\left\|\Phi_{\beta}(f)-\Phi_{\beta}^{\prime}(f) f\right\|_{L^{\infty}}
\end{array}
$$

3.3. Current estimate. Our first objective is an $L_{t, x}^{2}$ bound on the current $j_{\epsilon}=\frac{1}{\epsilon}\left(u_{\epsilon}-v_{\epsilon}\right)$.

Start from the relative entropy inequality (29) with the particular choice $\beta=\alpha$ (we recall that $\alpha$ is the exponent in the nonlinearity of the Carleman system (3) $)$.

We are going to show that, for $\gamma$ small enough, the term

$$
\frac{\gamma}{2} \int_{0}^{1} j_{\epsilon}(t, x)^{2} d x \text { is dominated by } \frac{1}{\epsilon^{2}} P_{\alpha}\left[u_{\epsilon}, v_{\epsilon}\right]
$$

Indeed, if $\alpha=1$

$$
\begin{aligned}
\frac{1}{\epsilon^{2}} P_{\alpha}\left[u_{\epsilon}, v_{\epsilon}\right] & =\frac{1}{\epsilon^{2}} \int_{0}^{1}\left(u_{\epsilon}^{2}-v_{\epsilon}^{2}\right)\left(\log u_{\epsilon}-\log v_{\epsilon}\right) d x \\
& =\int_{0}^{1} j_{\epsilon}^{2}\left(u_{\epsilon}+v_{\epsilon}\right) \frac{\log u_{\epsilon}-\log v_{\epsilon}}{u_{\epsilon}-v_{\epsilon}} d x \\
& \geq \int_{0}^{1} j_{\epsilon}^{2} \frac{u_{\epsilon}+v_{\epsilon}}{\max \left(u_{\epsilon}, v_{\epsilon}\right)} d x \geq \int_{0}^{1} j_{\epsilon}^{2} d x
\end{aligned}
$$

in view of the elementary inequality

$$
\frac{\log a-\log b}{a-b} \geq \frac{1}{\max (a, b)}, \quad \text { for each } a, b>0 .
$$


For $\alpha \in[0,1)$

$$
\begin{aligned}
\frac{1}{\epsilon^{2}} P_{\alpha}\left[u_{\epsilon}, v_{\epsilon}\right] & =\frac{1}{\epsilon^{2}} \int_{0}^{1}\left(u_{\epsilon}-v_{\epsilon}\right)\left(u_{\epsilon}+v_{\epsilon}\right)^{\alpha}\left(u_{\epsilon}^{1-\alpha}-v_{\epsilon}^{1-\alpha}\right) d x \\
& =\int_{0}^{1} j_{\epsilon}^{2}\left(u_{\epsilon}+v_{\epsilon}\right)^{\alpha} \frac{u_{\epsilon}^{1-\alpha}-v_{\epsilon}^{1-\alpha}}{u_{\epsilon}-v_{\epsilon}} d x \\
& \geq(1-\alpha) \int_{0}^{1} j_{\epsilon}^{2} \frac{\left(u_{\epsilon}+v_{\epsilon}\right)^{\alpha}}{\max \left(u_{\epsilon}, v_{\epsilon}\right)^{\alpha}} d x \geq(1-\alpha) \int_{0}^{1} j_{\epsilon}^{2} d x .
\end{aligned}
$$

Finally, for $\alpha \in[-1,0)$, we separate $(0,1)$ into the region where $u_{\epsilon}(t, x)>$ $v_{\epsilon}(t, x)$ and its complement, where $v_{\epsilon}(t, x) \geq u_{\epsilon}(t, x)$.

$$
\begin{aligned}
\frac{1}{\epsilon^{2}} P_{\alpha}\left[u_{\epsilon}, v_{\epsilon}\right] & =\frac{1}{\epsilon^{2}} \int_{0}^{1}\left(u_{\epsilon}-v_{\epsilon}\right)\left(u_{\epsilon}+v_{\epsilon}\right)^{\alpha}\left(u_{\epsilon}^{1-\alpha}-v_{\epsilon}^{1-\alpha}\right) d x \\
& =\int_{0}^{1} j_{\epsilon}^{2}\left(u_{\epsilon}+v_{\epsilon}\right)^{\alpha} \frac{u_{\epsilon}^{1-\alpha}-v_{\epsilon}^{1-\alpha}}{u_{\epsilon}-v_{\epsilon}} d x \\
& =\int_{u_{\epsilon}>v_{\epsilon}}+\int_{v_{\epsilon} \geq u_{\epsilon}} j_{\epsilon}^{2}\left(u_{\epsilon}+v_{\epsilon}\right)^{\alpha} \frac{u_{\epsilon}^{1-\alpha}-v_{\epsilon}^{1-\alpha}}{u_{\epsilon}-v_{\epsilon}} d x
\end{aligned}
$$

The first integral is estimated as follows:

$$
\begin{aligned}
\int_{u_{\epsilon}>u_{\epsilon}} j_{\epsilon}^{2}\left(u_{\epsilon}+v_{\epsilon}\right)^{\alpha} \frac{u_{\epsilon}^{1-\alpha}-v_{\epsilon}^{1-\alpha}}{u_{\epsilon}-v_{\epsilon}} d x & \geq \int_{u_{\epsilon}>v_{\epsilon}} j_{\epsilon}^{2}\left(2 u_{\epsilon}\right)^{\alpha} \frac{u_{\epsilon}^{1-\alpha}-v_{\epsilon}^{1-\alpha}}{u_{\epsilon}-v_{\epsilon}} d x \\
& =2^{\alpha} \int_{u_{\epsilon}>v_{\epsilon}} j_{\epsilon}^{2} \frac{u_{\epsilon}^{1+|\alpha|}-v_{\epsilon}^{1+|\alpha|}}{u_{\epsilon}^{1+|\alpha|}-u_{\epsilon}^{|\alpha|} v_{\epsilon}} d x \\
& \geq 2^{\alpha} \int_{u_{\epsilon}>v_{\epsilon}} j_{\epsilon}^{2} d x
\end{aligned}
$$

The second integral is estimated similarly. In all cases,

$$
\frac{1}{\epsilon^{2}} P_{\alpha}\left[u_{\epsilon}, v_{\epsilon}\right] \geq C_{\alpha} \int_{u_{\epsilon}>v_{\epsilon}} j_{\epsilon}^{2} d x
$$

with

$$
C_{1}=1 \text { while } C_{\alpha}=1-\alpha \text { if } 0 \leq \alpha<1 \text { and } C_{\alpha}=2^{\alpha} \text { if }-1 \leq \alpha<0 .
$$

Proposition 3.1. Let $\left(u_{\epsilon}, v_{\epsilon}\right)$ be a solution of the scaled, generalized Carleman system (3) with admissible initial and boundary data. Then there exists a positive constant $J \equiv J\left(\nu, \varphi_{m}, \alpha, T, u^{i n}, v^{i n}\right)>0$ such that the current $j_{\epsilon}=\frac{1}{\epsilon}\left(u_{\epsilon}-v_{\epsilon}\right)$ satisfies

$$
\int_{0}^{T} \int_{0}^{1} j_{\epsilon}^{2}(t, x) d x d t \leq J
$$

for each $\epsilon>0$. 
Proof. Start from (29), and pick $\gamma=C_{\alpha} / 2$. Upon integrating both sides of (29) over the time interval $[0, t]$, one obtains

$$
\begin{aligned}
& H_{\alpha}\left[u_{\epsilon}, v_{\epsilon} \mid f\right](t)+ \frac{3 C_{\alpha}}{4} \int_{0}^{t} \int_{0}^{1} j_{\epsilon}(s, x)^{2} d x d s \\
& \leq\left\|\partial_{t} \Phi_{\alpha}^{\prime}(f)\right\|_{L^{\infty}} \int_{0}^{t} H_{\alpha}\left[u_{\epsilon}, v_{\epsilon} \mid f\right](s) d s \\
& \quad+M_{0}\left(\nu, \alpha, \varphi_{m}\right) t+H_{\alpha}\left[u^{i n}, v^{i n} \mid f\right],
\end{aligned}
$$

where

$$
\begin{array}{r}
M_{0}\left(\nu, \alpha, \varphi_{m}\right) \geq \frac{1}{C_{\alpha}}\left\|\partial_{x} \Phi_{\alpha}(f)\right\|_{L^{\infty}}^{2}+\left\|\Phi_{\alpha}^{\prime \prime}(f) \partial_{t} f^{2}\right\|_{L^{\infty}} \\
+2\left\|\partial_{t} \Phi_{\alpha}^{\prime}(f)\right\|_{L^{\infty}}\left\|\Phi_{\alpha}(f)-\Phi_{\alpha}^{\prime}(f) f\right\|_{L^{\infty}}
\end{array}
$$

Gronwall's inequality implies that

$$
\begin{array}{r}
\left\|\partial_{t} \Phi_{\alpha}^{\prime}(f)\right\|_{L^{\infty}} \int_{0}^{t} H_{\alpha}\left[u_{\epsilon}, v_{\epsilon} \mid f\right](s) d s+M_{0}\left(\nu, \alpha, \varphi_{m}\right) t+H_{\alpha}\left[u^{i n}, v^{i n} \mid f\right] \\
\leq\left(M_{0}\left(\nu, \alpha, \varphi_{m}\right) t+H_{\alpha}\left[u^{i n}, v^{i n} \mid f\right]\right) e^{t\left\|\partial_{t} \Phi_{\alpha}^{\prime}(f)\right\|_{L^{\infty}}}
\end{array}
$$

from which we deduce that

$$
\int_{0}^{t} \int_{0}^{1} j_{\epsilon}(s, x)^{2} d x d s \leq \frac{4}{3 C_{\alpha}}\left(M_{0}\left(\nu, \alpha, \varphi_{m}\right) t+H_{\alpha}\left[u^{i n}, v^{i n} \mid f\right]\right) e^{t\left\|\partial_{t} \Phi_{\alpha}^{\prime}(f)\right\|_{L^{\infty}}}
$$

3.4. Density estimate. With the current estimate at our disposal, we next obtain an $L_{l o c}^{2}\left(\mathbf{R}_{+} ; L_{x}^{2}\right)$ bound on the macroscopic density $\rho_{\epsilon}=u_{\epsilon}+v_{\epsilon}$.

To do this, we apply (29) with $\beta=0$; here, the entropy production rate is useless so that we actually discard it from the left-hand side:

$$
\frac{d}{d t} H_{0}\left[u_{\epsilon}, v_{\epsilon} \mid f\right] \leq \frac{\gamma}{2} \int_{0}^{1} j_{\epsilon}(t, x)^{2} d x+\left\|\partial_{t} \Phi_{0}^{\prime}(f)\right\|_{L^{\infty}} H_{0}\left[u_{\epsilon}, v_{\epsilon} \mid f\right]+M_{1}(\nu, \gamma)
$$

where

$$
\begin{aligned}
M_{1}(\nu, \gamma) & \geq \frac{1}{2 \gamma}\left\|\partial_{x} f^{2}\right\|_{L^{\infty}}^{2}+\left\|\partial_{t} f^{2}\right\|_{L^{\infty}}+(1+\nu)^{2}\left\|\partial_{t} f\right\|_{L^{\infty}} \\
& \geq \frac{1}{2 \gamma}\left\|\partial_{x} \Phi_{0}(f)\right\|_{L^{\infty}}^{2}+\left\|\Phi_{0}^{\prime \prime}(f) \partial_{t} f^{2}\right\|_{L^{\infty}} \\
& +2\left\|\partial_{t} \Phi_{0}^{\prime}(f)\right\|_{L^{\infty}}\left\|\Phi_{0}(f)-\Phi_{0}^{\prime}(f) f\right\|_{L^{\infty}} .
\end{aligned}
$$

Integrating the relative entropy inequality above over the time interval $[0, t]$, we obtain

$$
\begin{aligned}
H_{0}\left[u_{\epsilon}, v_{\epsilon} \mid f\right](t) \leq H_{0}\left[u^{i n}, v^{i n} \mid f\right] & +\frac{\gamma}{2} J\left(\nu, \varphi_{m}, \alpha, t, u^{i n}, v^{i n}\right)+M_{1}(\nu, \gamma) t \\
& +\left\|\partial_{t} \Phi_{0}^{\prime}(f)\right\|_{L^{\infty}} \int_{0}^{t} H_{0}\left[u_{\epsilon}, v_{\epsilon} \mid f\right](s) d s .
\end{aligned}
$$

Gronwall's inequality implies that

$$
\begin{aligned}
& H_{0}\left[u_{\epsilon}, v_{\epsilon} \mid f\right](t) \\
& \leq\left(H_{0}\left[u^{i n}, v^{i n} \mid f\right]+\frac{\gamma}{2} J\left(\nu, \varphi_{m}, \alpha, t, u^{i n}, v^{i n}\right)+M_{1}(\nu, \gamma) t\right) e^{t\left\|\partial_{t} \Phi_{0}^{\prime}(f)\right\|_{L^{\infty}}}
\end{aligned}
$$


On the other hand,

$$
H_{0}\left[u_{\epsilon}, v_{\epsilon} \mid f\right](t)=\frac{1}{2}\left(\left\|u_{\epsilon}(t)-f(t)\right\|_{L_{x}^{2}}^{2}+\left\|v_{\epsilon}(t)-f(t)\right\|_{L_{x}^{2}}^{2}\right) .
$$

Summarizing the discussion above, we have proved the following density bound.

Proposition 3.2. Let $\left(u_{\epsilon}, v_{\epsilon}\right)$ be a solution of the scaled, generalized Carleman system (3) with admissible initial and boundary data. Then there exists a positive constant $K \equiv K\left(\nu, \varphi_{m}, \alpha, T, u^{i n}, v^{i n}\right)>0$ such that the macroscopic density $\rho_{\epsilon}=u_{\epsilon}+v_{\epsilon}$ satisfies

$$
\int_{0}^{T} \int_{0}^{1} \rho_{\epsilon}^{2}(t, x) d x d t \leq K
$$

for each $\epsilon>0$.

3.5. An entropy production estimate. We shall conclude this section with a further nonlinear estimate that eventually controls some flux in the diffusion limit.

We distinguish the cases $\alpha \in[0,1]$ and $\alpha \in[-1,0)$.

If $\alpha \in[0,1]$, we use (29) with $\beta= \pm 1$.

Indeed, the entropy production rate in the case $\beta=-1$ is

$$
\begin{aligned}
P_{-1}\left[u_{\epsilon}, v_{\epsilon}\right] & =\int_{0}^{1}\left(u_{\epsilon}+v_{\epsilon}\right)^{\alpha}\left(u_{\epsilon}-v_{\epsilon}\right)\left(\Phi_{-1}^{\prime}\left(u_{\epsilon}\right)-\Phi_{-1}^{\prime}\left(v_{\epsilon}\right)\right) d x \\
& =\int_{0}^{1}\left(u_{\epsilon}+v_{\epsilon}\right)^{\alpha}\left(u_{\epsilon}-v_{\epsilon}\right)\left(u_{\epsilon}^{2}-v_{\epsilon}^{2}\right) d x=\int_{0}^{1} \rho_{\epsilon}^{1+\alpha}\left(\epsilon j_{\epsilon}\right)^{2} d x .
\end{aligned}
$$

Hence the relative entropy inequality (29) with $\beta=-1$ becomes

$$
\begin{aligned}
\frac{d}{d t} H_{-1}\left[u_{\epsilon}, v_{\epsilon} \mid f\right]+\int_{0}^{1} \rho_{\epsilon}^{1+\alpha} j_{\epsilon}^{2} d x & \leq \frac{\gamma}{2} \int_{0}^{1} j_{\epsilon}(t, x)^{2} d x+M_{2}(\nu, \gamma) \\
& +\left\|\partial_{t} \Phi_{-1}^{\prime}(f)\right\|_{L^{\infty}} H_{-1}\left[u_{\epsilon}, v_{\epsilon} \mid f\right]
\end{aligned}
$$

with

$$
\begin{aligned}
M_{2}(\nu, \gamma) \geq & 2\left\|\partial_{t} \Phi_{-1}^{\prime}(f)\right\|_{L^{\infty}}\left\|\Phi_{-1}(f)-\Phi_{-1}^{\prime}(f) f\right\|_{L^{\infty}} \\
& +\frac{1}{2 \gamma}\left\|\partial_{x} \Phi_{-1}(f)\right\|_{L^{\infty}}^{2}+\left\|\Phi_{-1}^{\prime \prime}(f) \partial_{t} f^{2}\right\|_{L^{\infty}}
\end{aligned}
$$

Integrating the relative entropy inequality above in the time variable, and applying Proposition 3.1, we obtain

$$
\begin{aligned}
H_{-1}\left[u_{\epsilon}, v_{\epsilon} \mid f\right](t)+ & \int_{0}^{t} \int_{0}^{1} \rho_{\epsilon}^{1+\alpha} j_{\epsilon}^{2} d x \leq\left\|\partial_{t} \Phi_{-1}^{\prime}(f)\right\|_{L^{\infty}} \int_{0}^{t} H_{-1}\left[u_{\epsilon}, v_{\epsilon} \mid f\right](s) d s \\
& +H_{-1}\left[u^{i n}, v^{i n} \mid f\right]+\frac{\gamma}{2} J\left(\nu, \varphi_{m}, \alpha, T, u^{i n}, v^{i n}\right)+M_{2}(\nu, \gamma) t
\end{aligned}
$$

so that, by Gronwall's inequality, we arrive at an estimate of the form

$$
\begin{aligned}
& \int_{0}^{t} \int_{0}^{1} \rho_{\epsilon}^{1+\alpha} j_{\epsilon}^{2} d x \\
& \leq\left(H_{-1}\left[u^{i n}, v^{i n} \mid f\right]+\frac{\gamma}{2} J\left(\nu, \varphi_{m}, \alpha, T, u^{i n}, v^{i n}\right)+M_{2}(\nu, \gamma) t\right) e^{t\left\|\partial_{t} \Phi_{-1}^{\prime}(f)\right\|_{L^{\infty}}} .
\end{aligned}
$$


Doing the same with $\beta=1$ leads to

$$
\begin{aligned}
P_{1}\left[u_{\epsilon}, v_{\epsilon}\right] & =\int_{0}^{1}\left(u_{\epsilon}+v_{\epsilon}\right)^{\alpha}\left(u_{\epsilon}-v_{\epsilon}\right)\left(\ln \left(u_{\epsilon}\right)-\ln \left(v_{\epsilon}\right)\right) d x \\
& \geq \int_{0}^{1}\left(u_{\epsilon}+v_{\epsilon}\right)^{\alpha}\left(u_{\epsilon}-v_{\epsilon}\right) \frac{u_{\epsilon}-v_{\epsilon}}{u_{\epsilon}-v_{\epsilon}} d x=\int_{0}^{1} \rho_{\epsilon}^{\alpha-1}\left(\epsilon j_{\epsilon}\right)^{2} d x .
\end{aligned}
$$

The estimate above rests on the mean value theorem:

$$
(a-b)(\ln a-\ln b) \geq \frac{|a-b|}{\max (a, b)} \geq \frac{|a-b|}{a+b}, \quad a, b>0 .
$$

Integrating in $t$ the relative entropy inequality (29) with $\beta=1$ and applying the Gronwall inequality as above gives

$$
\begin{aligned}
& \int_{0}^{t} \int_{0}^{1} \rho_{\epsilon}^{\alpha-1} j_{\epsilon}^{2} d x \\
& \leq\left(H_{-1}\left[u^{i n}, v^{i n} \mid f\right]+\frac{\gamma}{2} J\left(\nu, \varphi_{m}, \alpha, T, u^{i n}, v^{i n}\right)+M_{3}\left(\nu, \varphi_{m}, \gamma\right) t\right) e^{t\left\|\partial_{t} \Phi_{-1}^{\prime}(f)\right\|_{L^{\infty}}},
\end{aligned}
$$

where

$$
\begin{aligned}
M_{3}\left(\nu, \varphi_{m}, \gamma\right) \geq & 2\left\|\partial_{t} \Phi_{1}^{\prime}(f)\right\|_{L^{\infty}}\left\|\Phi_{1}(f)-\Phi_{1}^{\prime}(f) f\right\|_{L^{\infty}} \\
& +\frac{1}{2 \gamma}\left\|\partial_{x} \Phi_{1}(f)\right\|_{L^{\infty}}^{2}+\left\|\Phi_{1}^{\prime \prime}(f) \partial_{t} f^{2}\right\|_{L^{\infty}} .
\end{aligned}
$$

If on the other hand $\alpha \in[-1,0)$, we use (29)) with $\beta=|\alpha|$. Notice that $\Phi_{|\alpha|}^{\prime \prime}$ is decreasing on $\mathbf{R}_{+}^{*}$ : indeed

$$
\begin{array}{ll}
\Phi_{|\alpha|}^{\prime \prime}(z)=(1-|\alpha|) z^{-|\alpha|} & \text { if } \alpha \in(-1,0), \\
\Phi_{|\alpha|}^{\prime \prime}(z)=z^{-1} & \text { if } \alpha=-1 .
\end{array}
$$

Hence, for each $a, b>0$, the mean value theorem implies that, for some $\theta \in(0,1)$

$$
(a-b)\left(\Phi_{|\alpha|}^{\prime}(a)-\Phi_{|\alpha|}^{\prime}(b)\right)=(a-b)^{2} \Phi_{|\alpha|}^{\prime \prime}((1-\theta) a+\theta b) \geq(a-b)^{2} \Phi_{|\alpha|}^{\prime \prime}(a+b) .
$$

Therefore

$$
\begin{aligned}
P_{|\alpha|}\left[u_{\epsilon}, v_{\epsilon}\right] & =\int_{0}^{1}\left(u_{\epsilon}+v_{\epsilon}\right)^{\alpha}\left(u_{\epsilon}-v_{\epsilon}\right)\left(\Phi_{|\alpha|}^{\prime}\left(u_{\epsilon}\right)-\Phi_{|\alpha|}^{\prime}\left(v_{\epsilon}\right)\right) d x \\
& \geq \int_{0}^{1}\left(u_{\epsilon}-v_{\epsilon}\right)^{2}\left(u_{\epsilon}+v_{\epsilon}\right)^{\alpha} \Phi_{|\alpha|}^{\prime \prime}\left(u_{\epsilon}+v_{\epsilon}\right) d x=C_{\alpha}^{\prime} \int_{0}^{1}\left(\epsilon j_{\epsilon}\right)^{2} \rho_{\epsilon}^{2 \alpha} d x,
\end{aligned}
$$

with

$$
C_{\alpha}^{\prime}=1 \text { if } \alpha=-1 \text { and } C_{\alpha}=1+\alpha \text { if } \alpha \in(-1,0) .
$$

The relative entropy inequality (29) with $\beta=|\alpha|$ becomes

$$
\begin{array}{r}
\frac{d}{d t} H_{|\alpha|}\left[u_{\epsilon}, v_{\epsilon} \mid f\right]+C_{\alpha} \int_{0}^{1} \rho_{\epsilon}^{2 \alpha} j_{\epsilon}^{2} d x \leq \frac{\gamma}{2} \int_{0}^{1} j_{\epsilon}(t, x)^{2} d x+M_{4}\left(\nu, \varphi_{m}, \gamma\right) \\
+\left\|\partial_{t} \Phi_{|\alpha|}^{\prime}(f)\right\|_{L^{\infty} H_{|\alpha|}}\left[u_{\epsilon}, v_{\epsilon} \mid f\right]
\end{array}
$$

with

$$
\begin{aligned}
M_{4}\left(\nu, \varphi_{m}, \gamma\right) \geq & 2\left\|\partial_{t} \Phi_{|\alpha|}^{\prime}(f)\right\|_{L^{\infty}}\left\|\Phi_{|\alpha|}(f)-\Phi_{|\alpha|}^{\prime}(f) f\right\|_{L^{\infty}} \\
& +\frac{1}{2 \gamma}\left\|\partial_{x} \Phi_{|\alpha|}(f)\right\|_{L^{\infty}}^{2}+\left\|\Phi_{|\alpha|}^{\prime \prime}(f) \partial_{t} f^{2}\right\|_{L^{\infty}}
\end{aligned}
$$


Integrating this inequality over the time interval $[0, t]$ and applying Gronwall's inequality and Proposition 3.1 as before, we obtain

$$
\begin{aligned}
& C_{\alpha} \int_{0}^{t} \int_{0}^{1} \rho_{\epsilon}^{2 \alpha} j_{\epsilon}^{2} d x \\
& \leq\left(H_{|\alpha|}\left[u^{i n}, v^{i n} \mid f\right]+\frac{\gamma}{2} J\left(\nu, \varphi_{m}, \alpha, T, u^{i n}, v^{i n}\right)+M_{4}\left(\nu, \varphi_{m}, \gamma\right) t\right) e^{t\left\|\partial_{t} \Phi_{|\alpha|}^{\prime}(f)\right\|_{L^{\infty}}} .
\end{aligned}
$$

Summarizing, we have proved

Proposition 3.3. Let $\left(u_{\epsilon}, v_{\epsilon}\right)$ be a solution of the scaled, generalized Carleman system (3) with admissible initial and boundary data. Then there exists a positive constant $L \equiv L\left(\nu, \varphi_{m}, \alpha, t, u^{i n}, v^{i n}\right)>0$ such that

$$
\begin{aligned}
& \int_{0}^{T} \int_{0}^{1} \rho_{\epsilon}^{\alpha+1} j_{\epsilon}^{2}(t, x) d x d t \leq L, \\
& \int_{0}^{T} \int_{0}^{1} \rho_{\epsilon}^{\alpha-1} j_{\epsilon}^{2}(t, x) d x d t \leq L
\end{aligned}
$$

for each $T>0$ and $\epsilon>0$ if $\alpha \in[0,1]$, and

$$
\int_{0}^{T} \int_{0}^{1} \rho_{\epsilon}^{2 \alpha} j_{\epsilon}^{2}(t, x) d x d t \leq L
$$

whenever $\alpha \in[-1,1]$.

In the case $\alpha \in[0,1]$, the second bound follows from the first and Proposition 3.1 by Hölder's inequality.

\section{The NONLINEAR DIFFUSION LIMIT}

Let $\left(u^{i n}, v^{i n}\right), \varphi^{+}$and $\varphi^{-}$be admissible initial and boundary data; then, for each $\epsilon>0$, let $\left(u_{\epsilon}, v_{\epsilon}\right)$ be the solution to the scaled, generalized Carleman system (3).

It follows from Propositions 3.1 and 3.2 that, for each $T>0$, one has

$$
\begin{gathered}
\left\|\rho_{\epsilon}\right\|_{L^{\infty}\left(0, T ; L^{2}(0,1)\right)} \leq K\left(\nu, \varphi_{m}, \alpha, T, u^{i n}, v^{i n}\right) \text { and } \\
\left\|j_{\epsilon}\right\|_{L^{2}([0, T] \times[0,1])} \leq J\left(\nu, \varphi_{m}, \alpha, T, u^{i n}, v^{i n}\right)
\end{gathered}
$$

for each $\epsilon>0$. By the Banach-Alaoglu theorem, for each $T>0$

the family $\rho_{\epsilon}$ is relatively compact in $L^{\infty}\left(0, T ; L^{2}(0,1)\right)$ weak-*

while

$$
\text { the family } j_{\epsilon} \text { is relatively compact in } L^{2}([0, T] \times[0,1]) \text { weak. }
$$

Summing both equations in Carleman's system implies that

$$
\partial_{t} \rho_{\epsilon}=-\partial_{x} j_{\epsilon}
$$

so that

$$
\partial_{t} \rho_{\epsilon} \text { is bounded in } L^{2}\left(0, T ; H^{-1}(0,1)\right) \text {; }
$$

with the bound on $\rho_{\epsilon}$ coming from Proposition 3.2, this control implies that

$$
\rho_{\epsilon} \text { is relatively compact in } C\left([0, T] ; H^{-1}(0,1)\right)
$$


by Arzela-Ascoli's theorem.

Likewise, since

$$
u_{\epsilon}=\frac{\rho_{\epsilon}+\epsilon j_{\epsilon}}{2} \text { and } v_{\epsilon}=\frac{\rho_{\epsilon}-\epsilon j_{\epsilon}}{2} \text {, }
$$

it follows that

$$
\begin{aligned}
& \partial_{x}\left(\rho_{\epsilon}+\epsilon j_{\epsilon}\right)=-2 \rho_{\epsilon}^{\alpha} j_{\epsilon}-\epsilon \partial_{t}\left(\rho_{\epsilon}+\epsilon j_{\epsilon}\right) \text { is bounded in } L^{2}\left([0,1] ; H^{-1}(0, T)\right) \\
& \partial_{x}\left(\rho_{\epsilon}-\epsilon j_{\epsilon}\right)=-2 \rho_{\epsilon}^{\alpha} j_{\epsilon}+\epsilon \partial_{t}\left(\rho_{\epsilon}-\epsilon j_{\epsilon}\right) \text { is bounded in } L^{2}\left([0,1] ; H^{-1}(0, T)\right)
\end{aligned}
$$

because of Propositions 3.1, 3.2 and 3.3. Hence

$$
\rho_{\epsilon} \pm \epsilon j_{\epsilon} \text { is relatively compact in } C\left([0,1] ; H^{-1}(0, T)\right)
$$

by Arzela-Ascoli's theorem.

Because on the nonlinearities that are present both in the scaled Carleman system (3) and in the limiting nonlinear diffusion equation (6), weak compactness results as above are not enough to pass to the limit as $\epsilon \rightarrow 0$. Strong $L^{2}$ compactness of the family $\rho_{\epsilon}$ is obtained by the same argument as in [10] (see also [9]), which we recall below.

Consider the vector fields

$$
p_{\epsilon}=\left(\rho_{\epsilon}, j_{\epsilon}\right) \text { and } q_{\epsilon}=\left(-\rho_{\epsilon}, \epsilon^{2} j_{\epsilon}\right) .
$$

By Propositions 3.1 and 3.2 , both vector fields satisfy

$$
p_{\epsilon} \text { and } q_{\epsilon} \text { are bounded in } L^{2}\left([0, T] \times[0,1] ; \mathbf{R}^{2}\right) \text {. }
$$

Summing the first two equations in the scaled Carleman system (3) shows that

$$
\operatorname{div}_{t, x} p_{\epsilon}=\partial_{t} \rho_{\epsilon}+\partial_{x} j_{\epsilon}=0
$$

while

$$
\operatorname{curl}_{t, x} q_{\epsilon}=\epsilon^{2} \partial_{t} j_{\epsilon}+\partial_{x} \rho_{\epsilon}=-2 \rho_{\epsilon}^{\alpha} j_{\epsilon}
$$

By Proposition 3.3, we therefore have

$$
\operatorname{div}_{t, x} p_{\epsilon} \text { and } \operatorname{curl}_{t, x} q_{\epsilon} \text { bounded in } L^{2}([0, T] \times[0,1]) .
$$

Pick any sequence $\epsilon_{n} \rightarrow 0$ such that

$$
\rho_{\epsilon_{n}} \rightarrow \rho \text { and } j_{\epsilon_{n}} \rightarrow j \text { in } L^{2}([0, T] \times[0,1])
$$

as $n \rightarrow \infty$. By compensated compactness (the div-curl lemma in [14]), we find that

$$
p_{\epsilon_{n}} \cdot q_{\epsilon_{n}}=-\rho_{\epsilon_{n}}^{2}+\epsilon_{n}^{2} j_{\epsilon_{n}}^{2} \rightarrow p \cdot q=\rho^{2}
$$

in the sense of Radon measures on $(0, T) \times(0,1)$ as $\epsilon_{n} \rightarrow 0$. Because of Proposition 3.1, this implies that

$$
\rho_{\epsilon_{n}}^{2} \rightarrow \rho^{2} \text { in the sense of Radon measures }
$$

which implies in turn that the family

$$
\rho_{\epsilon} \text { is relatively compact in } L^{2}([0, T] \times[0,1]) \text { strong. }
$$

Let then $(\rho, j, q)$ be a weak limit point in $L^{2}([0, T] \times[0,1])$ of the family $\left(\rho_{\epsilon}, j_{\epsilon}, \rho_{\epsilon}^{\alpha} j_{\epsilon}\right)$ as $\epsilon \rightarrow 0$ - the existence of such limits points being guaranteed 
by the bounds in Propositions 3.1, 3.2 and 3.3 together with the BanachAlaoglu Theorem.

Because of (33) and of the initial condition of the Carleman system (3), one has

$$
\rho \in C\left([0, T] ; H^{-1}(0,1)\right) \text { and }\left.\rho\right|_{t=0}=u^{i n}+v^{i n} .
$$

Because of (34) and of the boundary conditions of the scaled Carleman system (3), one has

$$
\rho \in C\left([0,1] ; H^{-1}(0, T)\right) \text { and }\left.\rho\right|_{x=0}=2 \varphi^{-}, \text {while }\left.\rho\right|_{x=1}=2 \varphi^{+} .
$$

Summing both equations in the scaled Carleman system shows that

$$
\partial_{t} \rho_{\epsilon}+\partial_{x} j_{\epsilon}=0
$$

so that, by passing to the limit in the relation above, one arrives at

$$
\partial_{t} \rho+\partial_{x} j=0
$$

in the sense of distributions on $\mathbf{R}_{+}^{*} \times(0,1)$.

Subtracting the second equation from the first in the scaled Carleman system shows that

$$
\epsilon^{2} \partial_{t} j_{\epsilon}+\partial_{x} \rho_{\epsilon}=-2 \rho_{\epsilon}^{\alpha} j_{\epsilon}
$$

so that, by passing to the limit in the equation above, one finds that

$$
\partial_{x} \rho=-2 q .
$$

Assume first that $\alpha \in[-1,0]$. By (35) and the weak-strong continuity of the product of two functions 2

$$
\rho^{|\alpha|} q=j
$$

so that

$$
j=-\frac{1}{2} \rho^{|\alpha|} \partial_{x} \rho=-\frac{1}{2(1-\alpha)} \partial_{x} \rho^{1-\alpha} .
$$

Substituting this in (38), we see that $\rho$ satisfies

$$
\begin{aligned}
& \partial_{t} \rho-\frac{1}{2(1-\alpha)} \partial_{x}^{2} \rho^{1-\alpha}=0, \\
& \rho(t, 0)=2 \varphi^{-}(t), \\
& \rho(t, 1)=2 \varphi^{+}(t), \\
& \rho(0, x)=u^{i n}(x)+v^{i n}(x) .
\end{aligned}
$$

Since this problem has a unique solution, the whole sequence $\rho_{\epsilon} \rightarrow \rho$ in $L^{2}([0, T] \times[0,1])$ as $\epsilon \rightarrow 0$, by the compactness statement in (35).

The case $\alpha \in(0,1)$ is slightly more delicate. Since $\rho \geq 0$ a.e. but may vanish, we set $\eta>0$ and, using as above the strong compactness statement (35) together with the weak-strong continuity of the product of two functions,

$$
\frac{1}{(\rho+\eta)^{\alpha}} q=\frac{\rho^{\alpha}}{(\rho+\eta)^{\alpha}} j
$$

\footnotetext{
${ }^{2}$ For $f: \mathbf{R}_{+} \rightarrow \mathbf{R}$ continuous and sublinear at infinity, if $\rho_{\epsilon} \rightarrow \rho$ in $L^{2}$ and $j_{\epsilon} \rightarrow j$ in $L^{2}, f\left(\rho_{\epsilon}\right) j_{\epsilon} \rightarrow f(\rho) j$ in $\mathcal{D}^{\prime}$.
} 
so that

$$
\frac{\rho^{\alpha}}{(\rho+\eta)^{\alpha}} j=-\frac{1}{2(1-\alpha)} \partial_{x}(\eta+\rho)^{1-\alpha} .
$$

We recall the argument in [9]. Letting $\eta \rightarrow 0$ shows that

$$
\mathbf{1}_{\rho>0} j=-\frac{1}{2(1-\alpha)} \partial_{x} \rho^{1-\alpha} .
$$

On the other hand, the second bound in Proposition 3.3 implies that

$$
\int_{0}^{T} \int_{0}^{1} \rho^{\alpha-1} j^{2} d x d t<+\infty
$$

so that

$$
j=0 \text { a.e. wherever } \rho=0 \text {. }
$$

Hence

$$
\mathbf{1}_{\rho>0} j=j
$$

so that the limiting nonlinear diffusion equation (39) also holds in the case $\alpha \in(0,1)$.

It only remains to treat the case $\alpha=1$. Proceeding as above, we obtain instead the relation

$$
\frac{\rho}{\rho+\eta} j=-\frac{1}{2} \partial_{x} \ln (\eta+\rho) .
$$

Integrating this relation in $x$ shows that

$$
\ln (\eta+\rho)(t, x)=\ln \left(\eta+\varphi^{+}(t)\right)-2 \int_{0}^{x} \frac{\rho}{\rho+\eta} j(t, z) d z
$$

so that, by Proposition 3.1 together with the bound $\varphi_{m} \leq \varphi^{+} \leq \nu$, we see that

$$
\int_{0}^{T} \int_{0}^{1} \sup _{\eta}|\ln (\eta+\rho)(t, x)| d t d x<+\infty .
$$

Hence, by dominated convergence, $\ln \rho \in L^{1}([0, T] \times[0,1])$ so that $\rho>0$ a.e. on $[0, T] \times[0,1]$. Therefore

$$
\frac{\rho}{\rho+\eta} j \rightarrow j \text { in } L^{2}([0, T] \times[0,1])
$$

and

$$
\ln (\eta+\rho) \rightarrow \ln \rho \text { in } L^{1}([0, T] \times[0,1])
$$

as $\eta \rightarrow 0$, so that

$$
j=-\frac{1}{2} \partial_{x} \ln \rho .
$$

Substituting this in (38), we see that $\rho$ satisfies

$$
\begin{aligned}
& \partial_{t} \rho-\frac{1}{2} \partial_{x}^{2} \ln \rho=0, \\
& \rho(t, 0)=2 \varphi^{-}(t), \\
& \rho(t, 1)=2 \varphi^{+}(t), \\
& \rho(0, x)=u^{i n}(x)+v^{i n}(x) .
\end{aligned}
$$


Again, since this problem has at most one solution, the whole sequence $\rho_{\epsilon}$ converges to that solution $\rho$ in $L^{2}([0, T] \times[0,1])$ because of the compactness statement (35).

Thus we have established the nonlinear diffusion limit for each $\alpha \in[-1,1]$.

\section{REFERENCES}

[1] T. Carleman. Problèmes mathématiques dans la théorie cinétique des gaz, Almqvist-Wiksells, Uppsala, 1957.

[2] D. Donatelli, P. Marcati. Convergence of singular limits for multi-D semilinear hyperbolic systems to parabolic systems, Trans. Amer. Math. Soc. 356 (2004) 20932121.

[3] W. E. Fitzgibion. The fluid-dynamical limit of the Carleman equation with reflecting boundary, J. Nonlin. Anal. Th. Meth. Appl. 6 (1982) 695-702.

[4] W. E. Fitzgibion. Initial-boundary value problem for the Carleman equation, Comput. Math. Appl. 9 (1983) 519-525.

[5] S. Goldstein. On diffusion by discontinuous movements, and on the telegraph equation, Quart. J. Mech. Appl. Math. 4 (1951) 129-156.

[6] F. Golse, F. Salvarani. Work in preparation.

[7] I. Kolodner. On Carleman's model for the Boltzmann equation and its generalizations, Ann. Mat. Pura Appl. 63 (1963) 11-32.

[8] T. G. Kurtz. Convergence of sequences of semigroups of nonlinear operators with an application to gas kinetics, Trans. Amer. Math. Soc. 186 (1973) 259-272.

[9] P. L. Lions, G. Toscani. Diffusive limits for finite velocities Boltzmann kinetic models, Rev. Mat. Iberoamericana, 13 (1997) 473-513.

[10] P. Marcati, A. J. Milani. The one-dimensional Darcy's law as the limit of a compressible Euler flow. J. Diff. Eq. 84 (1990) 129-147.

[11] P. Marcati, B. Rubino. Hyperbolic to parabolic relaxation theory for quasilinear first order systems, J. Differential Equations, 162 (2000) 359-399.

[12] H. P. MCKeAn. The central limit theorem for Carleman's equation, Israel J. Math. 21 (1975) 54-92.

[13] D. Mihalas, B. Weibel-Minalas. Foundations of Radiation Hydrodynamics Dover, Mineola NY, 1999.

[14] F. Murat. Compacité par compensation, Ann. Scuola Norm. Sup. Pisa Cl. Sci. 5 (1978) 489-507.

[15] A. Pulvirenti, G. Toscani. Fast diffusion as a limit of a two-velocity kinetic model, Rend. Circ. Mat. Palermo Suppl. 45 part II (1996) 521-528.

[16] R. Illner, M.C. Reed. The decay of solutions of the Carleman model. Math. Methods Appl. Sci. 3 (1981), 121-127.

[17] R. Illner, M.C. Reed. Decay to equilibrium for the Carleman model in a box. SIAM J. Appl. Math. 44 (1984), 1067-1075.

[18] F. SAlvarani. Diffusion limits for the initial-boundary value problem of the Goldstein-Taylor model. Rend. Sem. Mat. Univ. Politec. Torino 57 (1999) 209-220.

[19] F. Salvarani, J. L. VÁzquez. The diffusive limit for Carleman-type kinetic models. Nonlinearity 18 (2005) 1223-1248.

[20] L. TARTAR. Some existence theorems for semilinear hyperbolic systems in one space variable. Report \#2164, Mathematics Research Center, University of Wisconsin, Madison, 1980.

[21] G. I. TAYlor. Diffusion by continuous movements. Proc. London Math. Soc. 20 (1922) 196-212. 
F.G.: Université Paris 7 - Denis Diderot, Laboratoire J.-L. Lions, Boîte courrier 187, 4 Place Jussieu, 75252 Paris Cedex 05, France

F.S.: Dipartimento di Matematica, Università degli Studi di Pavia. Via Ferrata 1, 27100 PaVia, Italy 